\title{
IL CATALOGO DI DONNE DI VENZONE (SEC. XIV)
}

\section{PREMESSA}

Lo studio della scripta volgare tardomedievale consente, in generale, di acquisire importanti informazioni sulla storia delle varietà neolatine, una scripta che anticipa, molte volte, quei fenomeni e quelle strutture che vanno a costituire, con il tempo, i caratteri tipici delle singole varietà. Ciò è indubbiamente vero anche per il friulano, che possiede una cospicua tradizione manoscritta, tra XIV e XV secolo, soprattutto per quanto riguarda le scritture usuali, le carte di uso pratico. Si tratta di documenti, come minute di notai e di camerari, registri di comuni e di confraternite, che offrono importanti e anche vivaci testimonianze, in friulano, sulla vita delle comunità e sull'economia del tempo. Gli elementi di lessico comune che si possono isolare e studiare, con la lettura delle carte, sono numerosi, senza dubbio, ma ancora più numerosi sono gli antroponimi e i toponimi attestati nei documenti: se infatti i contesti e i tipi di operazioni riportate nelle carte si ripetono, anche di frequente, è l'onomastica invece a cambiare continuamente, con la costante segnalazione di nuovi luoghi e di nuove persone interessate alle singole operazioni.

Il problema del censimento di questo vasto patrimonio documentario, avvertito, si può dire, già dalla metà dell'Ottocento, pare finalmente vicino alla soluzione. Devo segnalare, a questo riguardo, i positivi risultati conseguiti dal pluriennale progetto di ricerca Documenti antichi dagli archivi friulani, diretto da chi scrive, avviato ormai nel febbraio del 2003. Si tratta, in particolare, di un progetto promosso dalla nostra Società Filologica Friulana di Udine in convenzione con il Ministero per i Beni culturali e con la Soprintendenza archivistica per il Friuli-Venezia Giulia, un progetto che prevedeva una ampia ricognizione, sistematica e tendenzialmente esauriente, dei fondi documentari antichi del Friuli storico fino alla fine del XV secolo. Nel primo triennio di lavoro, fino al febbraio del 2006, sono stati impegnati una ventina di collaboratori, tra archivisti e paleografi, e sono state prodotte circa 4.000 schede catalografiche, rivolte alla descrizione di archivi (enti conservatori ed enti produttori), fondi, serie e singoli documenti di interesse. Contestualmente alla ricognizione degli archivi, sono stati segnalati e trascritti un cospicuo numero di documenti di uso pratico in volgare friulano. Nel marzo del 2006, per presentare i risultati del progetto, si è tenuto a Udine un convegno di studi, gli atti del quale sono stati recentemente pubblicati sul primo numero del 2007 [ma: marzo 2009]

\footnotetext{
* Indirizzo dell'autore: Dipartimento di Lingue e Letterature germaniche e romanze, Via Mantica 3, 33100 Udine, Italia. Email: federico.vicario@uniud.it / federico.vicario@tele2.it
} 
della rivista Rassegna degli Archivi di Stato. ${ }^{1}$ Ci si augura, naturalmente, di poter riprendere al più presto il lavoro, bloccato in attesa della stipula di una nuova convenzione, auspicando anche un maggiore coinvolgimento della Regione autonoma Friuli-Venezia Giulia, in particolare della Direzione degli archivi, Regione che è chiamata ad assumere un ruolo centrale nel complesso sistema di conservazione e di valorizzazione dei beni culturali del nostro territorio. ${ }^{2}$

In questo contributo, che con vero piacere offro al prof. Mitja Skubic, insigne romanista e appassionato studioso dei fenomeni di contatto tra il mondo romanzo e il mondo slavo, mi propongo di riproporre e di commentare un documento del Trecento proveniente da Venzone, località a pochi chilometri da Gemona del Friuli, un documento ricco di spunti relativi al lessico e all'onomastica antica del Friuli.

\section{IL CATALOGO DI DONNE DI VENZONE DEL XIV SECOLO}

La Biblioteca civica di Udine, erede del Museo cittadino, conserva collezioni manoscritte di straordinario interesse per la storia e per la cultura friulana. Grazie ad un impegnativo programma di ricognizione dei fondi della Biblioteca e di pubblicazione dei documenti antichi in volgare friulano, condotto negli ultimi dieci anni, vd. Vicario (1999, in particolare la Premessa, pp. 13-17, per la presentazione del lavoro), disponiamo di un complesso di edizioni che al momento, quanto meno per ampiezza, in Friuli non ha pari. Nel corso degli anni, in particolare, è stato quindi pubblicato un registro del 1382 della Confraternita di Santa Maria dei Battuti di Udine, che si occupava dell'amministrazione dell'ospedale cittadino, un quaderno di cameraria della Pieve di Tricesimo, per gli anni 1426-1437, i cospicui rotoli della Confraternita dei Calzolai di Udine, dei primi del Quattrocento, in cinque volumi - quattro contenenti l'edizione delle carte e il quinto con il glossario e gli indici onomastici - e infine tre volumi miscellanei di carte antiche, a partire dalla fine del XIII secolo, di varia provenienza e dimensioni. ${ }^{3}$ Nell'ultimo di questi volumi, quello del 2008 (pp. 145-152), ho presentato anche una pergamena del XIV secolo, l'Elenco di iscritte a un pio sodalizio di Venzone. $\mathrm{Si}$ tratta del secondo manoscritto venzonese della Biblioteca civica di Udine, per

\footnotetext{
${ }^{1}$ La pubblicazione, cui naturalmente si rimanda, contiene la presentazione del progetto e i suoi risultati, vd. Vicario (2007a), una serie di articoli su singoli fondi documentari o aree della regione e, infine, una breve rassegna di testi friulani antichi segnalati nel corso della ricerca, vd. Vicario (2007b).

${ }^{2}$ La segnalazione e la descrizione paleografica dei documenti tardomedievali in volgare non esaurisce il compito di chi si proponga di portare un contributo allo studio del friulano antico, ma senza dubbio si pone come punto di partenza fondamentale per lo sviluppo di un programma di pubblicazione dei documenti stessi. Solo a partire dalla costituzione di un ampio repertorio di forme antiche si può passare, infatti, alla redazione di opere di ampia prospettiva diacronica, come un vocabolario storico, un dizionario dei nomi personali, una storia della lingua.

3 Previsti in uscita nel 2009 sono gli indici lessicali e onomastici relativi a questi tre ultimi volumi pubblicati.
} 
ampiezza, il primo essendo stato pubblicato, nel 1874, da Alexander Wolf, un cospicuo inventario di redditi della locale confraternita di Santa Maria dei Battuti. ${ }^{4}$

La pergamena, della quale riproponiamo qui la trascrizione, con un paio di marginali interventi all'edizione e con commento linguistico, è legata all'interno del quarto volume della raccolta delle Pergamene Friulane, un volume che raccoglie documenti, non numerati, dal 1393 al 1406. ${ }^{5}$ L'intitolazione Catalogo di donne di Venzone probabilmente iscritte in un pio sodalizio è da attribuirsi a Vincenzo Joppi, come anche la datazione del pezzo al XIV secolo. Di questo documento, interessante soprattutto per l'onomastica personale, si sono occupati anche Giovanni Battista Corgnali (1934), per la discussione, in particolare, del formante diminutivale -ùs e -ùz per il femminile, e Gianfranco D'Aronco (1982, pp. 77-81). ${ }^{6}$ La pergamena è perfettamente regolare e piegata in quattro, con facciate da mm. $315 \mathrm{x}$ 230 (stesa misura mm. 315 x 450), ed era utilizzata come coperta di un fascicolo d'archivio, appartenente ai conti Antonini: ciò si desume da una nota del 1682, che compare sull'ultima facciata del fascicolo stesso. ${ }^{7}$ L'elenco è vergato da più mani, come si può rilevare dalla variazione degli stessi tipi onomastici e lessicali all'interno del testo, ed è quasi sicuramente mutilo, dal momento che comincia senza alcuna intestazione; Corgnali suppone, tra l'altro, che esso dovesse comprendere anche gli Statuti della fraternita di Santa Maria dei Battuti di Venzone, che sono però andati perduti.

Si è aggiunta, nell'edizione, la numerazione delle righe, assente sul manoscritto. Il Catalogo comprende in tutto 219 nomi, disposti su due colonne; nell'edizione si va a capo ad ogni riga, numerate sul margine destro della pagina, e si segnala, in nota, il cambio di colonna. Le parole vergate unite nell'originale sono state separate secondo l'uso moderno, con il trattino orizzontale, quando si ha elisione di vocale; anche le maiuscole sono state segnate secondo l'uso moderno. Le note all'edizione sono riportate in numeri romani alla fine di ogni carta. Sono stati aggiunti, infine, alcuni accenti tonici (gravi) per facilitare la lettura e la comprensione del testo.

\footnotetext{
4 Pubblicato integralmente, con revisioni e integrazioni all'edizione, il manoscritto è ora presentato in Vicario (2006-08, vol. II, pp. 13-59) e commentato, dal punto di vista linguistico, in Vicario (2008a), lavoro al quale si rimanda anche per una presentazione, più generale, dei fondi manoscritti di Venzone.

${ }^{5} \mathrm{La}$ raccolta delle Pergamene friulane corrisponde al ms. 1231 del Fondo Principale della Biblioteca e questo quarto volume, in particolare, al ms. 1231/4.

6 Il Catalogo di donne era stato segnalato al Corgnali dallo stesso Joppi, cui si deve materialmente anche la raccolta delle Pergamene friulane; questo pezzo, nonostante il suo indubbio interesse, non viene però inserito dallo studioso di Tarcento nella sua nota antologia dei Testi inediti friulani, che pubblica nel 1878, con commento linguistico di Graziadio Isaia Ascoli, sul quarto numero dell'Archivio Glottologico Italiano.

${ }^{7}$ La raccolta delle Pergamene friulane corrisponde al ms. 1231 del Fondo Principale della Biblioteca e questo quarto volume, in particolare, al ms. 1231/4.
} 
c. $1 \mathrm{r}$

Katerina del Mulin ${ }^{\mathrm{i}}$

Domenia Pilot

Chumina la Schusarie ${ }^{\mathrm{ii}}$

Uliana Ingistalt

Catarina di Simon Mian

Madalena Merlot

La baia Baldiçar

Piç mogleriii Cristoful sartor

Blasiola Baçeit

La Quochula

Crofessa

Francescha mogler Stefin

Madalena Taragnot

Vignuda Filit

Tomasina di Indriùs

Margaretta Corat

Madalena di Sant Çorç

Vignudìs di Nichulau Paculin

Madalena Fiçot

Cataruç Stolf

La Iostanella

Provencia Savi

La mogler Vidiço

Nuse Chichan

Margaretta Rodonger

Uliùs Babòs

La Marchiole

Vignut Pagisin

Iustina di Samonç

Chiliana

Catarina Laçarin

Gitùs monica di santa Maria

La Uçilutta

Galdrut dicta Pirlùsiv

Cataruç di Iacum Coç

Gnisùs Galuç

Viniria

Vignuda di Simon Indrich

Ursula Martin fari

Galdrut di Pontaleon

40

Margaretta Cont

Cuminuç Mulaiç 
Madalena Coset

Sabida mogler Fidrich

Madalena di Lenart carador

La mogler Iosef fari

Catari[n]a Candin Pitilin

Catarina Pançeir

Chuchuluç Scorset

La Scorsetta

La mogler Candon

Flor Tomat Coç

Aricafina

Betta Claboch

Margiritùs di Iacum da Ridolf

Francescha Sant

Cataruç Prinel

Madalena Çichut

Vignudaç

Dona Ursula

60

Margaretta Uanegar

Gitùs Uischang

Catarina Pacet

Anussa mogler Benedet

Birtulina di Pluorn

Catarina neca Canduc

i colonna di sinistra

ii anche Corgnali, vergato però Schusaric

iii vergato mol con abbreviazione; in seguito anche mogler e muglir per esteso

iv colonna di destra

c. $1 \mathrm{v}$

Clara la Çuanella ${ }^{\mathrm{i}}$

Luca Bidot

Margeretta mogler Lorenç

La Blaudine ${ }^{\text {ii }}$

Margaretta Micul Bos

Gnesa Dot

Tussa Vigliset

Dona Nicholosa

Vignuda di Iacum Martin

La Missetta

La Tarnabane

Vignuda figle Zuan Baffe 
Cataruç Çaçarel

Lena mogler Dinel Marabeç

Lena la monia

Lorença Candòs

Dona Baltrama

Madalena mogler Martin Broilet

Claruç mogler Çorç Toschan

Catarina Trapan

La Biçuita

Catarina $^{\text {iii }}$ mogler Niculau Maurìs ${ }^{\text {iv }}$

Betta Lenardin

Dona Lena

Kataruç del Michìs

Mos mogler Petri del Ost

Sabida la Masile

Birtulina di mestri March

Mancilina

Vignuda Pigurin

Francescha Fiçot

Palmuç Fuçùs

Dona Biatrìs di Pertistan

La mogler Bertul Rix

Margaretta mogler Andrea Pilot ${ }^{\mathrm{V}}$

Silia di Iacum Philipan

Marta Biata ${ }^{\text {vi }}$

Dona Cirincina

Margaretta Canduç

Catarina mogler Çorç di Samonç

Catarina la Bergine

Vignuda Matio Broilet

Dorotea di Moç

Katarina Cuchuluç

Vignuda mogler Durich Todesch

Culussa Iacum Scriç

La rimita di sant Iacum

Margaretta Pitul

Milena

Catarina Uargent

Niculusse Michel dela Nera

Çuana di Bortolomio Poç

Madalena mogler Siart Meçinbrout

Birtulina Pieri Iaculg 
Margaretta di Mena

La mogler Iançil del Lion

Vignuda Muy

Galiana

Miniùs mogler Bortolot 1-Ugulinvii

Tonie figlie del Çot

Vinta mogler di Pieri Carnel

Margaretta Gnesan

Dona Simona di Antony Poç

La Çotta Minot

Katerina ${ }^{\text {viii }}$ la mogler di Simon Vignut

Menia di Niculau cartar

La mogler Iacum dell[a] Plaça

i colonna di sinistra

ii si corregge qui la lettura proposta in Vicario (2006-08, vol. I, p. 148)

iii con $t a$ nell'interlineo superiore

iv vergato Maris con abbreviazione

v colonna di destra

vi Corgnali antepone un dona che non c'è

vii con segno di abbreviazione superfluo

viii aggiunto dalla stessa mano sulla stessa riga

c. $2 \mathrm{r}^{\mathrm{i}} \quad$ Çuanina Pilirin mulinar ${ }^{\mathrm{ii}}$

La mogler Indriùs Piçul

Madalene de N. Pieri Menìs

Catarina la Zacha[...] de Mozo

Madalena de Madùs de Resia

Subette mogler cu fo Pieri Zanpel

Agite di Iachum Zunchunin

Sigidie Iacum dela Stella

Bortholomee Iacum Pugnet

Ursule mogler cu fo de Pieri Cichut

Lena mogler che ${ }^{\mathrm{iii}}$ fo Niculau Catùs ${ }^{\mathrm{iv}}$

Leonarde muglir Martin de Sant Denel

Lene mogler che fo Francesch de Sequals

Catarina mogler Zuan Daç

Lena Tarvisan

La mogler Murilv de Ovedàs

Fracescha de Bone d-Amar

Madalùs figle Burtulùs de Quall

Lucia sorella Lazer de Moço 
Sabide di Pieri del Blanch

Culusse mogler Toni Simot d-Amar

Margareta de Pieri Pusan

Miniùs de Andrea di Peglonis

Clare mogler Zuan Munyti de Portis

Zuanina mogler Pichull de Samonç

Paschuta de Forglarie

Maria de Mation Marcagno

Palma mogler Benedet de Inpeç

Birtulina mogler ser Durì de ser Aitor d-Udin

La Pascula

La mogler Nicolò Feret

Simone mogler Zuan tesedor

Miniùs mogler Stiefin Caristia de Tarnep

Catarina figle Zorç Canduçvi

Gnese Pedròs de Resia

Vignude de Buia

Benvignude de Simon Paculin

Ursule mogler Uliff syridurar

Margareta de Toni vichary

Birtuline de Mazoll de Mozo

Catarine de Simon de Andrea

Margareta de Andrea batafer

Tonie figle Zuan de Pers

Margareta de Niculau Zamul

Ursule de Bortolot Radiff

Anne figle de Domeny Minigin

Catarine de Lenart Tertin

Menie de Zuan de Zara

Ursule figle de Zuan tessedor

La moniga de sant Iacum

Lena mogler de ser Nicolò Paculin

Piran de Domenìs Marabeç

Chatarina la Moltesa de Mozo

La mogler Blas Flor

Aligranze mogler Andrea Candin

Uliana de Iacum Varagnut

Filipa mogler Bortolot Bareit ${ }^{v i i}$

Stella d-Andrea Marcuç

Sabida de Iacumin de Çulg

Margareta figle Culau Preoles

Margareta Poç

Catarine de Domeni de Alnitviii 
Vignude mogler Zuan Pacet

Catarine mogler Tomat < zudio > de Mozo

Culussa Simot de Amar

Margareta figla che fo ser Çorzo Pitacul

Margareta la maistra dicta Cantula

Çuana che fo mogler Culau Milene

Flandina di Pilirin de Cavaç

Catarina de Domeni Silimon

Brida mogler Culau Tomadot

Aligranza Laurinzut de Amar

Cuza de Zuan Razulin de Cavaç

i frequenti cambi di mano

ii colonna di sinistra

iii vergato ch con abbreviazione; troviamo per esteso, per il medesimo contesto, sia cu che che

iv Corgnali legge, o interpreta, Cacus

v Corgnali legge Anuril con punto di domanda

vi colonna di destra

vii Corgnali legge bazeit

viii sic

c. 2v Margareta mogler Stiefin Starnaç ${ }^{\mathrm{i}}$

Dorothea mogler Lebisorch

Catarina mogler Culau Monet

La mogler Mation Candin

Margareta de Simon Pacet de Ovedàs

Marie ${ }^{i i} \operatorname{mog}[1]$ er Indrea Paurin

Chostanza muglir Nichulau mestry Zuan

Barbire fig[1]e Simion Vignut

Bete de Fantùs

Francescha mogler Lenart Saladin

Beta mogler Toni Chiapilin de Cavaç

Bortolamea monia de sancta Maria

Lena moier che fo Cullau Martinuzo

i unica colonna, a sinistra

ii lettura incerta anche con la lampada di Wood; Corgnali mette Aurie 


\section{NOTE AL TESTO}

\subsection{Gli antroponimi}

Le tre principali categorie di antroponimi, che troviamo nel nostro Catalogo, sono quelle consuete dei nomi personali (o individuali), dei nomi aggiunti e dei soprannomi; il numero complessivo dei nomi, per le tre categorie, è abbastanza rilevante, trovandosene anche tre o quattro per ogni riga della pergamena.

Fanno parte della prima classe di nomi, i personali o individuali, gli appellativi della tradizione latina, germanica, greca, cristiana, o di origine augurale, forme che stanno alla base di numerosissimi nomi propri e cognomi friulani moderni. ${ }^{8}$ L'elenco di tipi che qui brevemente si propone comprende anche numerosi derivati, con la presenza di alcune forme di diminutivo, soprattutto, con i suffissi -UTTUS, -ITTUS, -INUS, -USS(I)US, -ELLUS, -UCIUS, di accrescitivo, in -ONE, -OTTUS, -ATTUS, quest'ultimo talora anche con valore dispregiativo. I diminutivi, come prevedibile, risultano particolarmente numerosi; ciò si deve anche al fatto che nel Catalogo prevalgono i nomi femminili, che spesso, con il diminutivo, sottolineano la grazia e la gentilezza della persona che li porta, doti che si sogliono attribuire alle donne. Molto diffusi sono anche gli ipocoristici, cioè le forme abbreviate dei nomi stessi, di norma per aferesi della sillaba atona iniziale.

Tra i nomi tradizionalmente più diffusi nella regione friulana troviamo qui alcuni continuatori di 'Giovanna', con Çuana e le due forme di diminutivo Çuanella e Çuanina; il nome corrispondente al maschile 'Nicola, Nicolao' è rappresentato dalla forma di diminutivo Niculusse, anche ipocoristico Culusse, e dall'accrescitivo Nicholosa. Per 'Giacoma, Giacomina', altro nome in genere molto diffuso, abbiamo invece i due ipocoristici Chumina e Cuminuç, quest'ultimo con doppio suffisso diminutivo; 'Domenica' presenta la regolare forma friulana Domenia, con dileguo della velare intervocalica, e gli ipocoristici Menia, Miniùs, quest'ultimo al diminutivo. 'Caterina', un nome solo femminile, si registra con la forma primaria Katerina o Catarina e il diminutivo Cataruç.

Particolarmente numerosi per i nomi femminili, come si diceva, sono i diminutivi. Abbiamo Gnisùs da Gnesa 'Agnese', Anussa 'Annetta', Vignudis da Vignuda 'Benvenuta', entrambi ipocoristici di Benvignude, che si trova anche nella forma Vignudaç, con suffisso dispregiativo, Birtulina da 'Berta', Blasiola 'Biagetta', Iustina 'Giustina', Madalùs da Madalena 'Maddalena', Marchiole per 'Marcolina', Margitùs e Gitùs da Margaretta 'Margherita', Paschuta da 'Pasqua' e Tomasina da 'Tommasa';

\footnotetext{
${ }^{8}$ Il processo di fissazione di vere e proprie forme cognominali, nel Friuli del Tre e Quattrocento, non è però ancora compiuto; nel caso dei patronimici e dei matronimici tale processo pare, però, più avanzato rispetto ad altri tipi. Per un'analisi storica ed etimologica degli appellativi qui proposti, si rimanda comunque al fondamentale lavoro di Rossebastiano/Papa (2005), anche se non sempre, in realtà, i tipi friulani equivalgono o corrispondono precisamente a quelli italiani. Per una presentazione generale dell'onomastica personale friulana si rimanda a Frau (1988); altri lavori, su singoli pezzi o raccolte, sono proposti da Vicario (2008b) e (2008c).
} 
nella forma Tussa, infine, possiamo vedere una forma breve per Bertussa o per Margitussa. Altri nomi personali, questa volta senza diminutivo, sono poi Agite 'Agata', Aligranze 'Allegranza', Baltrama 'Bertanda', Betta 'Elisabetta', Biatris 'Beatrice', Bortholomee e Bortolamea 'Bartolomea', Chostanza 'Costanza', Clara 'Chiara', Dorotea 'Dorotea', Filipa 'Filippa', Flor 'Fiora', Francescha 'Francesca', Galdrut 'Geltrude', Galiana 'Galliana', Lena per 'Elena' o 'Maddalena', Leonarde 'Leonarda', Lorença 'Lorenza', Lucia 'Lucia', Maria 'Maria', Marta 'Marta', Milena 'Milena', Palma 'Palma', Sabida 'Sabata', Silia 'Marsilia', Simona 'Simona', Stella 'Stella', Subette 'Alzubetta', Tonie 'Antonia', Uliana 'Giuliana', Ursula e Ursule 'Orsola', Viniria 'Veneria'.

Molti e vari sono anche i nomi maschili, nel nostro Catalogo; si tratta, in genere, dei nomi dei padri, dei mariti o dei fratelli delle donne che vi sono indicate. Numerosi sono, in particolare, gli appellativi di origine germanica, alcuni decisamente desueti, appellativi che hanno avuto grande fortuna e circolazione nel Friuli patriarcale. Abbiamo, in ogni caso: Aitor 'Ettore', Antony 'Antonio', anche con l'ipocoristico Toni, Baldiçar 'Baldassarre', Benedet 'Benedetto', Blas 'Biagio', Bortolomio 'Bartolomeo', Bortolot e Burtulùs da 'Berto', Corat 'Corrado', Çorç 'Giorgio', Cristoful 'Cristoforo', Dinel 'Daniele', Domeni 'Domenico', con i diminutivi Domenis, Menis e Minigin, Durì e Durich 'Odorlico', Fidrich 'Federico', Francesch 'Francesco', anche al diminutivo Çichut, Iacum 'Giacomo', Indrea 'Andrea', Indrich 'Enrico', con il diminutivo Indriùs, il germanico Ingistalt, che non risulta avere corrispondenti in italiano, Iosef 'Giuseppe', Lazer 'Lazzaro' e il diminutivo Laçarin, Lenart 'Leonardo', anche con il diminutivo Lenardin, Lorenç 'Lorenzo', con il diminutivo Laurinzut, con conservazione del dittongo, Luca 'Luca', il diminutivo Madùs per 'Amedeo', March 'Marco' e il suo diminutivo Marcuç, Martin 'Martino' e il diminutivo Martinuzo, Matio 'Matteo' con l'accrescitivo Mation, Mian 'Damiano', Michel 'Michele', Nichulau 'Nicolao, Nicolò', con il comune ipocoristico Culau, Philipan 'Filippo', Pieri 'Pietro', con Pedròs, Pilirin 'Pellegrino', Pontaleaon 'Pantaleone', Ridolf 'Rodolfo', il raro Siart 'Sigheardo', Simion 'Simeone' e Simon 'Simone', Stiefin e Stefin 'Stefano', Stolf 'Astolfo', Tomat 'Tommaso', con l'accrescitivo Tomadot, Vignut 'Benvenuto' e, infine, Zuan 'Giovanni', con la variante Iançil, di tradizione germanica.

Le categorie dei nomi aggiunti e dei soprannomi comprendono gli appellativi forse più interessanti e curiosi, tra quelli che si registrano nel Catalogo, appellativi entrati, di norma, dal lessico friulano comune e attribuiti per molti e diversi motivi, talora oscuri. Tra questi, scorrendo la lista, si segnala il comune Pilot (r. 2), frl. pilot 'verrettone, freccia', a definire una persona alta, magra o nervosa; abbiamo poi questa Chumina la Schusarie (r. 3) 'Giacomina la Sbucciatrice, la Scortecciatrice', dal frl. scus$s \hat{a}$ 'sbucciare, scorzare, escoriare', ad indicare una persona che aiutava in un mulino o in un'altra manifattura. Il Merlot della r. 6 è il 'merlo comune', il Vidiço della r. 23 richiama il frl. vidice, vidiçon 'tralcio della vite', a significare magari la passione o l'abuso di vino. Asciutta o con la voce chiara sarà la Uçilutta 'Uccellina' della r. 33, mentre Galdrut dicta Pirlùs, della riga successiva, richiama il frl. pirlâ 'girare come una trottola', anche pirli 'trottola', quindi 'Geltrude detta Trottolina', di persona sempre 
indaffarata. Non molto alti e slanciati saranno stati Iacum Coç (r. 35) e Tomat Coç (r. 52), dal frl. comune coç 'corto', e minuto anche il Pitilin 'piccolo' della r. 47. Dal frl. scorsâ 'rincorrere, cacciare via', anche scorse 'rincorsa, corsa', abbiamo il maschile Scorset, alla r. 49, e il femminile Scorsetta, alla riga successiva, probabilmente la moglie del suddetto Scorset. La Blaudine della r. 70 potrebbe essere il frl. comune blaudin 'vitalba', una pianta arbustiva piuttosto diffusa, come il Bos della riga successiva il frl. bos 'bosso comune'; la Missetta di r. 76 era mediatrice di affari o di matrimoni, cfr. frl. missete 'sensale', Zuan Baffe, alla riga 78, aveva forse passione per la buona cucina, cfr. frl. bafe 'cotenna del maiale, lardo'. Sabida la Masile, r. 93, aveva forse una mascella pronunciata, e la Marta Biata di r. 103 non era probabilmente molto pronta e sveglia, cfr. frl. biât 'beato', ma anche 'sciocco'. Altri nomi aggiunti, che richiamano il lessico comune, sono Cuchuluç (r. 110), frl. cocule 'noce', Scriç (r. 112), frl. scriç 'scricciolo, uccellino', Poç (r. 118), frl. poç 'pozzo', Lion (r. 122), frl. leon, lion 'leone' (sempre che non si tratti però di un ipocoristico di Pontaleon, vd. sopra), Çot (r. 126) e Çotta (r. 130), frl. çuet 'zoppo', Piçul (r. 135), frl. piçul 'piccolo', Zunchunin (r. 140), frl. çonc 'pezzo di tronco', Pugnet (r. 142), frl. pugn 'pugno', Catùs (r. 144), frl. catùs 'assiolo, chiù, civetta', anche 'sciocco, scimunito', Blanch (r. 153), frl. blanc 'bianco', Feret (r. 164), frl. fier 'ferro', Caristia (r. 166), frl. cjaristie 'carestia', e altri ancora.

A fianco del nome personale e del patronimico, concorre talvolta all'identificazione di una certa persona anche l'indicazione della professione, del mestiere, della funzione. ${ }^{9}$ Tra questi elementi, anch'essi molto produttivi nella formazione dei cognomi friulani moderni, segnaliamo: baia 'balia, nutrice', frl. baie; batafer 'fabbro ferraio, battiferro', frl. batifier; carador 'carrettiere, barrocciaio', frl. cjaradôr; cartar 'cartaio', frl. cjartâr; fari 'fabbro', frl. fari; mestri 'mastro, operaio', con il femminile maistra, frl. mestri; monia, moniga e monica 'suora', frl. muinie; mulinar 'mugnaio', frl. mulinâr; rimita 'eremita', frl. rimit, remit; sartor 'sarto', frl. sartôr; syridurar 'serraturaio, magnano, fabbro', cfr. frl. mod. sieradure; tesedor 'tessitore', frl. tiessidôr; vichary 'vicario, vice, facente funzioni', frl. vicjari.

\subsection{I toponimi e gli etnici}

Frequente nell'identificazione di una certa persona, insieme al riferimento al nome dei genitori o del mestiere, è l'indicazione del luogo di provenienza. Nel nostro Catalogo i toponimi non sono poi così numerosi, in realtà, ma ci consentono comunque di rilevare una serie di località nelle vicinanze di Venzone (ma il toponimo Avençon o Vençon non è mai registrato, probabilmente perché la maggior parte delle persone erano proprio di lì e quindi l'indicazione sarebbe risultata superflua), della Carnia e del Friuli centrale. ${ }^{10}$ Nelle vicinanze della cittadina abbiamo quindi le locali-

9 Una essenziale rassegna di tali elementi, tratti dallo Schedario onomastico di Giovanni Battista Corgnali, è tracciato in Frau/Marcato (1997).

10 Alcuni toponimi sono presenti più volte, nel documento: si indica, qui, solo la prima attestazione di ogni singolo tipo. 
tà di Pluorn 'Pioverno di Venzone' (r. 65), con conservazione della nasale scoperta in fine di parola (cfr. frl. mod. Pluver), di Portis 'Portis di Venzone' (r. 157), con monottongo (cfr. frl. mod. Puartis) e di Samonç 'Sottomonte di Venzone' (r. 29). Numerose sono le località della Carnia e del Canal del Ferro, con Amar 'Amaro' (r. 150), Cavaç 'Cavazzo Carnico' (r. 202), Çulg 'Zuglio' (r. 192), Inpeç 'Ampezzo' (r. 161), Moç 'Moggio Udinese' (r. 109), ancora una volta con monottongo (cfr. frl. mod. Mueç), anche Moço (r. 152), con restituzione della vocale finale, con la frazione di Ovedàs 'Ovedasso di Moggio Udinese' (r. 149), e quindi Peglonis 'Peonis di Trasaghis' (r. 156), Resia 'Resia' (r. 138) e Tarnep 'Interneppo di Bordano' (r. 166).

Oltre alla città di Udin 'Udine' (r. 162), in area più centrale abbiamo ancora Buia 'Buja' (r. 169), Forglarie 'Forgaria nel Friuli' (r. 159), Pers 'Pers di Majano' (r. 176), Pertistan 'Partistagno di Attimis' (r. 99), Sant Denel 'San Daniele del Friuli' (r. 145) e, oltre il Tagliamento, Sequals 'Sequals' (r. 146). Registriamo inoltre due toponimi comuni, come Alnit 'Alneto, bosco di alni' (r. 195) e Quall 'Colle' (r. 151), che, senza maggiori indicazioni, potrebbero ancora riguardare il territorio di Venzone, come anche Sant Çorç 'San Giorgio' (r. 17), anch'esso piuttosto diffuso. Fuori dal Friuli abbiamo, alla fine, Zara 'Zara' (r. 181), ora in Croazia.

Derivati dai toponimi, per indicare la provenienza, sono gli aggettivi etnici. ${ }^{11}$ La categoria non è qui particolarmente rappresentata; abbiamo infatti solo Tarnabane 'di Interneppo (Bordano)', Tarvisan forse 'di Tarvisio', piuttosto che 'di Treviso', Toschan 'toscano', Todesch 'tedesco', e l'immancabile Carnel 'carnico, originario della Carnia'.

\section{OSSERVAZIONI CONCLUSIVE}

Per concludere questa essenziale presentazione del Catalogo di donne di Venzone del XIV secolo, esempio certo interessante di scripta friulana delle origini e fonte per lo studio dell'onomastica locale, non mi resta che ribadire l'importanza del sistematico esame dei documenti tardomedievali di uso pratico della nostra regione. I lavori di ricognizione dei fondi e il programma di edizioni dei manoscritti di interesse, in corso ormai da alcuni anni, hanno portato a risultati che consentono di avviare, con fiducia, la redazione di un repertorio, ampio e affidabile, di forme antiche. Questo lavoro, forse non breve e nemmeno facile, non pare comunque avere alternative alla sua realizzazione, se si vogliono intraprendere quelle opere lessicografiche e storiche che ancora mancano nel panorama di studi sul friulano. Mi riferisco in primo luogo ad un dizionario comprensivo delle fonti tardomedievali, così notevoli e abbondanti, ma poi anche ad un repertorio dell'onomastica personale.

Costituisce un modello e un sicuro riferimento, a questo proposito, quello che rimane ancora oggi il principale strumento per lo studio dell'antroponimia di area

11 Molto documentato e ricco di informazioni è, a questo proposito, il recente saggio di Marcato/Puntin (2008), che supera ampiamente gli studi precedenti. 
friulana, il monumentale e inedito Schedario Onomastico di Giovanni Battista Corgnali, ricco di circa 160.000 schedine manoscritte e conservato presso la Biblioteca Civica di Udine. Fa piacere segnalare che è già in corso dai primi mesi del 2009, a cura dello scrivente e con la collaborazione di alcuni giovani ricercatori, la digitalizzazione integrale dello Schedario su database informatico in rete, con il controllo e la segnalazione delle fonti a suo tempo utilizzate dal Corgnali. A cinquanta e più anni dalla morte del suo autore, magnifico esempio di laboriosità e di tenacia, ma anche di competenza e di dedizione nell'illustrazione del patrimonio linguistico e culturale del Friuli, i tempi paiono maturi per promuovere l'aggiornamento e il completamento, con l'apporto di quanto prodotto in questi decenni, anche di questo straordinario repertorio.

\section{Bibliografia}

Corgnali, Giovanni Battista (1934) «Onomastica friulana. Del diminutivo femminile in -ùs e in -ùz.» Ce fastu? 14, 103-107.

Corgnali, Giovanni Battista (1965-67) «Scritti e testi friulani (cur. Gaetano Perusini).» Ce fastu? 41-43, i-x, 1-405.

D'Aronco, Gianfranco (1982) Nuova antologia delle letteratura friulana. Dalle origini al Settecento. Vol. I. Udine: Ribis.

FraU, Giovanni (1988) «Per la storia dei cognomi friulani.» Atti dell'Accademia di Scienze, Lettere e Arti di Udine 81, 247-263.

FraU, Giovanni/Marcato, Carla (1997) «Antichi nomi di mestieri nel Patriarcato di Aquileia (Italia nordorientale) e loro riflessi storico-linguistici.» Memorie Storiche Forogiuliesi 77, 75-90.

JopPI, Vincenzo (1878) «Testi inediti friulani dei secoli XIV al XIX.» Archivio Glottologico Italiano 4, 185-342.

Marcato, Carla/Puntin, Maurizio (2008) Etnici e blasoni popolari nel Friuli storico. Udine: Società Filologica Friulana.

Marchetti, Giuseppe (1934) «Studi sulle origini del friulano. L'onomastica.» Ce fastu? 10, 87-93.

Piccini, Daniela (2006) Lessico latino medievale in Friuli. Udine: Società Filologica Friulana.

Pirona, Giulio Andrea/Ercole Carletti/Giovanni Battista Corgnali (1935) Il Nuovo Pirona, Vocabolario friulano. [Con aggiunte e correzioni riordinate da Giovanni Frau, 21992]. Udine: Società Filologica Friulana.

Rossebastiano, Alda/Papa, Elena (2005) I nomi di persona in Italia. Dizionario storico ed etimologico. 2 voll. Torino: UTET.

VICARIO, Federico (1999) Il quaderno dell'Ospedale di Santa Maria Maddalena. Udine: Biblioteca Civica.

VICARIO, Federico (2000) Il quaderno della Fraternita di Santa Maria di Tricesimo. Udine: Biblioteca Civica.

VICARIO, Federico (2001-05) I rotoli della Fraternita dei Calzolai di Udine. 5 voll. Udine: Biblioteca Civica. 
VICARIO, Federico (2006) «Fonti documentarie tardomedievali e studi lessicografici sul friulano.» In: F. Bruni/C. Marcato (a cura di), Lessicografia dialettale. Ricordando Paolo Zolli. Roma/Padova: Antenore, 189-200.

VICARIO, Federico (2006-08) Carte friulane antiche dalla Biblioteca Civica di Udine. 3 voll. Udine: Biblioteca Civica.

VICARIO, Federico (2007a) «Documenti antichi dagli archivi friulani. Il progetto.» Rassegna degli Archivi di Stato n. s. 3/1, 19-31.

VICARIO, Federico (2007b) «Edizione di documenti in volgare friulano tra XIII e XV secolo.» Rassegna degli Archivi di Stato n. s. 3/1, 100-120.

VICARIO, Federico (2008a) «Appunti di antroponimia friulana da un inventario di redditi del sec. XV.» In: G. Blaikner-Hohenwart et alii (a cura di), Ladinometria. Festschrift für Hans Goebl zum 65. Geburtstag. Lavis (Tn): Universität Salzburg, 363-375.

VICARIO, Federico (2008b) «Note di antroponimia tergestina.» In: G. Arcamone (a cura di), Atti del XXII Congresso Internazionale di Scienze Onomastiche. Pisa: ETS, 823-834.

VICARIO, Federico (2008c) «Note di onomastica da carte gemonesi del Trecento.»In: P. Lendinara/S. Serafin (a cura di), Un tuo serto di fiori in man recando. Scritti in onore di Maria Amalia D'Aronco. Vol. I. Udine: Forum, 291-299.

Wolf, Alexander (1874) «Un testo friulano dell'anno 1429.» Annali scientifici del Regio Istituto Tecnico di Udine 7, 3-27.

\section{Riassunto \\ IL CATALOGO DI DONNE DI VENZONE (SEC. XIV)}

Nell'articolo si presenta l'edizione di un documento friulano tardomedievale, il Catalogo di donne di Venzone (sec. XIV), un documento conservato presso la Biblioteca Civica di Udine «V. Joppi». All'edizione, diplomatica, segue un commento linguistico, in particolare sull'onomastica personale, sul lessico comune e sulla toponomastica; l'esame del testo consente di isolare numerosi elementi di interesse, nomi femminili e maschili, ma anche nomi di mestiere e funzione, che costituiscono gli antecedenti di numerosi cognomi moderni.

Si conferma l'importanza fondamentale dello studio sistematico delle carte friulane di uso pratico, come quelle qui presentate, operazione preliminare e indispensabile al fine di avviare, in seguito, opere di lessicografia di ampia prospettiva, in primis la redazione di un dizionario storico comprensivo delle forme antiche. Sembra quest'ultimo un obiettivo ormai alla portata, potendo contare sui progressi che la disciplina ha compiuto negli ultimi anni, con lo svolgimento di un vasto progetto di ricognizione dei fondi archivistici di tutto il Friuli storico promosso dalla Società Filologica Friulana e dal Ministero per i Beni culturali (Documenti antichi dagli archivi friulani). Ai positivi risultati ottenuti con lo svolgimento di questo progetto si aggiungono le numerose edizioni realizzate su fondi manoscritti conservati in alcuni degli enti conservatori più importanti della regione, a partire ancora dai materiali antichi depositati presso la Biblioteca Civica di Udine. 


\section{Povzetek}

\section{IL CATALOGO DI DONNE DI VENZONE (14. STOL.)}

$\mathrm{V}$ članku je predstavljena izdaja poznosrednjeveškega furlanskega dokumenta Il catalogo di donne di Venzone (14. stol.), ki ga hrani Videnska mestna knjižnica »V. Joppi«. Diplomatski izdaji sledi jezikoslovni komentar, predvsem o osebnem imenoslovju, o splošnem besedišču in o toponomastiki. V besedilu je mogoče najti številne zanimive elemente, in sicer ženska in moška imena, pa tudi poimenovanja poklicev in funkcij, iz katerih izhajajo mnogi sodobni priimki.

Iz gradiva se potrjuje temeljna pomembnost sistematičnega proučevanja furlanskih dokumentov pragmatične narave, kakršni so tukaj predstavljeni. Takšno proučevanje pomeni namreč nepogrešljivo začetno opravilo, ki nam omogoča, da se nato lotimo obsežnih leksikografskih projektov, predvsem zgodovinskega slovarja, ki bi vključeval tudi stare oblike. Ta cilj se sedaj zdi že na dosegu roke, saj je mogoče računati na napredek, ki ga je stroka dosegla v zadnjih letih z obsežnim projektom popisa in pregleda arhivskih fondov na območju celotnega zgodovinskega ozemlja Furlanije, katerega pobudnik sta Furlansko filološko društvo in Ministrstvo za kulturno dediščino (naslov projekta: DOCUMENTI ANTICHI DAGLI ARCHIVI FRIULANI). Pozitivnim rezultatom tega projekta se pridružujejo številne izdaje, pripravljene na osnovi rokopisnih fondov iz nekaterih najpomembnejših furlanskih arhivskih ustanov, med katerimi je treba spet omeniti stare dokumente iz Videnske mestne knjižnice. 